\title{
ELECTROHYDRODYNAMIC THERMAL INSTABILITY IN A WALTERS' (MODEL B') ROTATING NANOFLUID SATURATING A POROUS MEDIUM
}

\author{
Gian C. Rana ${ }^{1 *}$, Poonam K. Gautam ${ }^{2}$, Hemlata Saxena ${ }^{2}$ \\ ${ }^{1}$ Department of Mathematics, NSCBM Govt. College, Hamirpur-177 005, Himachal Pradesh, \\ India \\ e-mail: drgcrana15@gmail.com \\ ${ }^{2}$ Department of Mathematics, Career Point University, Kota, Rajasthan, India \\ e-mail: poornima17gautam@gmail.com \\ *corresponding author
}

\begin{abstract}
The onset of thermal instability in a dielectric rotating nanofluid layer saturating a porous medium with vertical AC electric field is investigated by employing Darcy model for porous medium. The rheology of the nanofluid is described by Walters' (model B') for calculating the shear stresses from the velocity gradients. The employed model incorporates the combined effects of movement of the molecules of the fluid striking the nanoparticles, thermophoresis and electrophoresis due to the embedded particles. The boundaries are considered to be stress free. It is assumed that nanoparticle flux is zero on the boundaries. The eigen-value problem is solved analytically using the first approximation of Galerkin method. The Darcy Rayleigh number for onset of nonoscillatory (stationary) modes is obtained. The effects of the modified Taylor number, the AC electric Rayleigh number, the Lewis number, the modified diffusivity ratio, nanoparticles Rayleigh number and medium porosity have been discussed. The kinematic viscoelasticity accounting for rheology of the nanofluid has no effect on the stationary convection for Walters' (model B') nanofluids and behaves like an ordinary Newtonian nanofluid. Oscillatory convection has been ruled out under the considered boundary conditions.
\end{abstract}

Keywords: nanofluid, AC electric field, Rayleigh number, Walters' (model B'), electrohydrodynamic, Brownian motion, Galerkin method

\section{Introduction}

Electrohydrodynamics (EHD) finds diverse applications in enhancement of thermal transfer, EHD pumps, micromechanic systems, micro-cooling systems, building of thermal insulation, biomechanics etc. Electrohydrodynamic thermal instability in a porous medium is a phenomenon related to various fields. It has various applications in different areas such as EHD enhanced thermal transfer, EHD pumps, EHD in microgravity, micromechanic systems, drug delivery, micro-cooling system, nanotechnology, oil reservoir modeling, petroleum industry, building of thermal insulation, biomechanics, engineering etc. Chandrasekhar (1961) has given a 
comprehensive account of thermal instability of Newtonian fluid under the various assumptions of hydrodynamics and hydromagnetics. The investigation in porous media has been started with the Darcy model. A good account of convection problems in a porous medium is given in Ingham (1981), Vafai and Hadim (2000) and Nield and Bejan (2006). Electrodynamics of continuous media and electrohydrodynamic convection in fluids has been studied by Landau, (1960), Roberts (1969) and Castellanos (1998). Electrohydrodynamics is a branch of fluid mechanics which deals with the motion of fluid under the influence of electrical forces. It can also be considered as that part of electrodynamics which is necessitated with the influence of moving media on electric fields. Electrohydrodynamics involves both the effect of fluid in motion and the influence of the field in motion (Melcher et al. 1969; Jones 1978). Nanofluid was first coined by Choi (1995). Further, suspensions of nanoparticles are being developed for medical applications including cancer therapy. The detailed study of thermal convection in a layer of nanofluid in porous medium based upon Buongiorno (2006) model has been discussed by different authors (Tzou 2008a, b; Nield and Kuznetsov 2009; Sheu 2011; Chand and Rana 2012; Nield and Kuznetsov 2014; Chand et al. 2014; Yadav and Kim 2015; Chand et al. 2017; Chand and Rana 2017).

All the studies referred above deal with Newtonian nanofluids. However, with the growing importance of non-Newtonian fluids in geophysical fluid dynamics, chemical technology and petroleum industry attracted widespread interest in the study on non-Newtonian nanofluids. One such type of fluids is Walters' (model B') (1962) elastico-viscous fluid having relevance in chemical technology and industry. Walters' (model B') elastico-viscous fluid forms the basis for the manufacture of many important polymers and useful products. A good account of thermal instabilities problems in a Walters' (Model B') elastico-viscous fluid in a porous medium is given in Sharma and Rana (2001), Gupta and Aggarwal (2011), Shivakumara et al. (2011) and Rana et al (2012).

Recently, considerable interest has been evidenced in the study of electrohydrodynamic thermal instability in viscous and viscoelastic fluid. Takashima (1976) discussed the effect of uniform rotation on the onset of convective instability in a dielectric fluid under the simultaneous action of AC electric field. The onset of electrohydodynamic instability in a horizontal layer of viscous and viscoelastic fluid was studied by Takashima and Ghosh (1979), Takashima and Hamabata (1984), Othman (2004), Shivakumara et al. (2007), Ruo et al. (2010), Shivakumara et al. (2013) and Rana et al. $(2015,16)$.

The growing number of applications of electrohydrodynamic thermal instability in an elasticoviscous nanofluid in a porous medium which include several engineering and medical fields, such as automotive industries, energy saving and cancer therapy, motivated the current study. Our main aim is to study the effect of vertical AC electric field on the onset of thermal instability in a horizontal layer of an elastico-viscous Walters' (Model B') nanofluid under rotation in a porous medium.

\section{Formulation of the problem and mathematical model}

Here we consider an infinite horizontal porous layer of a Walters' (model B') elasticoviscous rotating nanofluid of thickness $d$, bounded by the planes $\mathrm{z}=0$ and $z=d$ and subject to a uniform vertical AC electric field applied across the layer; the lower surface is grounded and the upper surface is kept at an alternating $(60 \mathrm{~Hz})$ potential whose root mean square value is $\mathrm{V}_{1}$ (see Fig.1). The layer is heated from below, which is acted upon by a gravity force $g=(0,0,-g)$ aligned in the $z$ direction. The temperature, $T$, and the volumetric fraction of nanoparticles, $\varphi$, at the lower (upper) boundary is assumed to take constant values $T_{0}$, and $\varphi_{0}\left(T_{1}\right.$, and $\left.\varphi_{1}\right)$, respectively. We know that keeping a constant volume fraction of nanoparticles at the horizontal boundaries will be almost impossible in a realistic situation. However, we assumed these 
conditions, which have also been previously adopted by several authors (Tzou 2008 a, b; Nield and Kuznetsov 2009; Sheu 2011; Chand and Rana 2012; Nield and Kuznetsov 2014; Chand et al. 2014; Yadav and Kim 2015; Takashima and Ghosh 1979; Takashima and Hamabata 1984; Othman 2004; Shivakumara et al. 2007; Ruo et al. 2010; Shivakumara et al. 2013; and Rana et al. 2015, 2016, Chand et al. 2017 and Chand and Rana 2017).

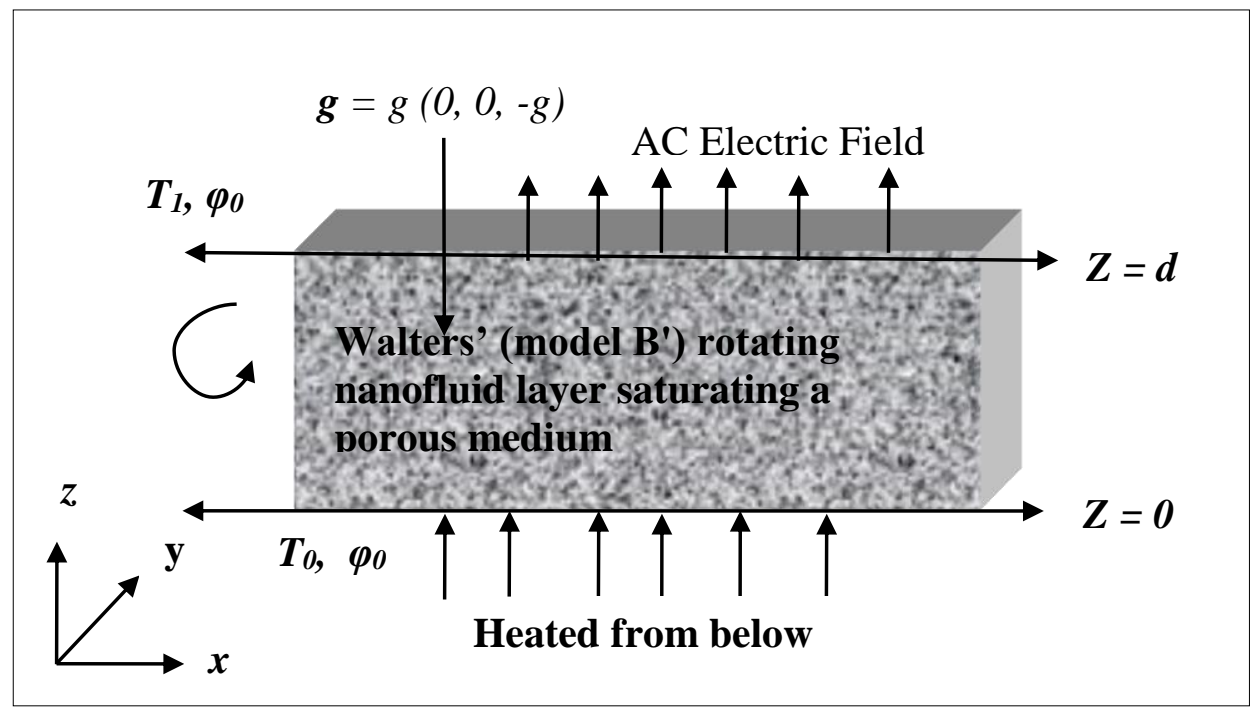

Fig. 1. Physical Configuration

\subsection{Governing Equations}

The equations of mass-balance and momentum-balance for Walters' (model B') elastico-viscous with vertical AC electric field (Chandrasekher 1961, Tzou 2008a, b, Nield and Kuznetsov 2009, Sheu 2011, Chand and Rana 2012, Nield and Kuznetsov 2014, Chand et al. 2014, Yadav and Kim 2015, Chand et al. 2017, Chand and Rana 2017) under the Oberbeck-Boussinesq approximation in a porous medium are

$$
\begin{gathered}
\nabla \cdot \mathbf{q}=0, \\
\frac{\rho}{\varepsilon}\left[\frac{\partial \mathbf{q}}{\partial t}+\frac{1}{\varepsilon}(\mathbf{q} \cdot \nabla) \mathbf{q}\right]=-\nabla P+\rho \mathbf{g}-\frac{1}{k_{1}}\left(\mu-\mu^{\prime} \frac{\partial}{\partial t}\right) \mathbf{q}+2 \frac{\rho}{\varepsilon}(\mathbf{q} \times \mathbf{\Omega})-\frac{1}{2}(E \cdot E) \nabla K,
\end{gathered}
$$

Where

$$
\mathrm{P}=p-\frac{\rho}{2} \frac{\partial K}{\partial \rho}(\mathbf{E} \cdot \mathbf{E})
$$

is the modified pressure (Takashima 1976) and $\rho, \mu, \mu, p, \varepsilon, \mathrm{k}_{1}, \boldsymbol{E}, K, \mathbf{q}(\mathrm{u}, \mathrm{v}, \mathrm{w})$, denote respectively, density, viscosity, viscoelasticity, pressure, medium porosity, medium permeability, root mean square value of the electric field and Darcy velocity vector, respectively. The $\rho$ density of the nanofluid can be written (Buongiorno 2006) as

$$
\rho=\varphi \rho_{p}+(1-\varphi) \rho_{f}
$$


where $\varphi$ is the volume fraction of nanoparticles, $\rho_{p}$ is the density of nanoparticles and $\rho_{f}$ is the density of base fluid. We approximate the density of the nanofluid by that of the base fluid, that is we consider $\rho=\rho_{f}$ (Tzou 2008a, b; Nield and Kuznetsov 2009; Sheu 2011; Chand and Rana 2012). Now, introducing the Boussinesq approximation for the base fluid, the specific weight, $\rho g$ in equation (2) becomes

$$
\rho \mathbf{g} \cong\left(\varphi \rho_{p}+(1-\varphi)\left\{\rho\left(1-\alpha\left(T-T_{0}\right)\right)\right\}\right) \mathbf{g}
$$

where $\alpha$ is the coefficient of thermal expansion.

If one introduces a buoyancy force, the equation of motion for Walters' (model B') nanofluid by using Boussinesq approximation and Darcy model for porous medium (e.g. Nield and Kuznetsov 2009) is given by

$$
0=-\nabla P+\left(\varphi \rho_{\mathrm{p}}+(1-\varphi)\left\{\rho\left(1-\alpha\left(\mathrm{T}-\mathrm{T}_{0}\right)\right)\right\}\right) \mathbf{g}-\frac{1}{\mathrm{k}_{1}}\left(\mu-\mu^{\prime} \frac{\partial}{\partial t}\right) \mathbf{q}+2 \frac{\rho}{\varepsilon}(\mathbf{q} \times \mathbf{\Omega})-\frac{1}{2}(E \cdot E) \nabla K
$$

The mass-balance equation for the nanoparticles (Buongiorno 2006) is

$$
\frac{\partial \varphi}{\partial t}+\frac{1}{\varepsilon} \mathbf{q} \cdot \nabla \varphi=D_{B} \nabla^{2} \varphi+\frac{D_{T}}{T_{0}} \nabla^{2} T .
$$

The thermal energy equation for a nanofluid is

$$
(\rho \mathrm{c})_{\mathrm{m}}\left[\frac{\partial \mathrm{T}}{\partial \mathrm{t}}+\mathbf{q} \cdot \nabla \mathrm{T}\right]=\mathrm{k}_{\mathrm{m}} \nabla^{2} \mathrm{~T}+\varepsilon(\rho \mathrm{c})_{\mathrm{p}}\left(\mathrm{D}_{\mathrm{B}} \nabla \varphi \cdot \nabla \mathrm{T}+\frac{\mathrm{D}_{\mathrm{T}}}{\mathrm{T}_{0}} \nabla \mathrm{T} \cdot \nabla \mathrm{T}\right)
$$

where $(\rho c)_{m}$ is heat capacity of fluid in porous medium, $(\rho c)_{p}$ is heat capacity of nanoparticles and $k_{m}$ is thermal conductivity.

The Maxwell equations are

$$
\begin{gathered}
\nabla \times \mathbf{E}=0 \\
\nabla \cdot(K \mathbf{E})=0
\end{gathered}
$$

Let $\mathrm{V}$ be root mean square value of electric potential, the electric potential can be expressed as

$$
\mathbf{E}=-\nabla V
$$

The dielectric constant is assumed to be linear function of temperature and is of the form

$$
K=K_{0}\left[1-\gamma\left(T-T_{0}\right)\right]
$$

where $\gamma>0$, is the thermal coefficient of expansion of dielectric constant and is assumed to be small.

We assume that the temperature is constant and nanoparticles flux is zero on the boundaries. Thus, boundary conditions $(1,18)$ are

$$
\begin{aligned}
& w=0, T=T_{0}, D_{B} \frac{\partial \phi}{\partial z}+\frac{D_{T}}{T_{0}} \frac{\partial T}{\partial z}=0 \text { at } z=0 \\
& \text { and } w=0, T=T_{1}, D_{B} \frac{\partial \phi}{\partial z}+\frac{D_{T}}{T_{1}} \frac{\partial T}{\partial z}=0 \text { at } z=d
\end{aligned}
$$


We introduce non-dimensional variables as

$$
\begin{aligned}
& \left(\mathrm{x}^{\prime}, \mathrm{y}^{\prime}, \mathrm{z}^{\prime},\right)=\left(\frac{\mathrm{x}, \mathrm{y}, \mathrm{z}}{\mathrm{d}}\right),\left(\mathrm{u}^{\prime}, \mathrm{v}^{\prime}, \mathrm{w}^{\prime},\right)=\left(\frac{\mathrm{u}, \mathrm{v}, \mathrm{w}}{\kappa_{\mathrm{m}}}\right) \mathrm{d}, \mathrm{t}^{\prime}=\frac{\mathrm{t} \kappa}{\sigma \mathrm{d}^{2}}, \\
& \mathrm{P}^{\prime}=\frac{\mathrm{Pk}_{1}}{\mu \kappa_{\mathrm{m}}}, \varphi^{\prime}=\frac{\varphi-\varphi_{0}}{\varphi_{1}-\varphi_{0}}, \mathrm{~T}^{\prime}=\frac{\mathrm{T}-\mathrm{T}_{1}}{\mathrm{~T}_{0}-\mathrm{T}_{1}}, \mathrm{~K}^{\prime}=\frac{\mathrm{K}}{\gamma \mathrm{E}_{0} \Delta T d},
\end{aligned}
$$

where $\kappa_{\mathrm{m}}=\frac{\mathrm{k}_{\mathrm{m}}}{\left(\rho \mathrm{c}_{\mathrm{P}}\right)_{\mathrm{f}}}$ is thermal diffusivity of the fluid and $\sigma=\frac{\left(\rho c_{P}\right)_{m}}{\left(\rho \mathrm{c}_{\mathrm{P}}\right)_{\mathrm{f}}}$ is the thermal capacity ratio. We obtain the equations in non-dimensional form (after dropping the dashes ( ' ) for convenience) as

$$
\begin{gathered}
\nabla \cdot \mathbf{q}=0, \\
0=-\nabla P-\left(1-F \frac{\partial}{\partial t}\right) \mathbf{q}-R m \hat{e}_{z}+R a T \hat{e}_{z}-R n \varphi \hat{e}_{z}+\sqrt{T a}\left(v \hat{e}_{x}-\hat{e}_{y}\right)+R_{e a} \nabla_{H}^{2}\left(T-\frac{\partial K}{\partial z}\right), \\
\frac{1}{\sigma} \frac{\partial \varphi}{\partial t}+\frac{1}{\varepsilon} \mathbf{q} \cdot \nabla \varphi=\frac{1}{L e} \nabla^{2} \varphi+\frac{N_{A}}{L e} \nabla^{2} T, \\
\frac{\partial T}{\partial t}+\mathbf{q} \cdot \nabla T=\nabla^{2} T+\frac{N_{A}}{L e} \nabla \varphi \cdot \nabla T+\frac{N_{A} N_{B}}{L e} \nabla T \cdot \nabla T, \\
\nabla^{2} V=\frac{\partial T}{\partial z}
\end{gathered}
$$

$\begin{array}{ll}L e=\frac{\kappa_{m}}{D_{B}} & \text { is the thermal Lewis number, } F=\frac{\mu \kappa_{m}}{\mu \sigma d^{2}} \text {, is the kinematic visco-elasticity } \\ \text { where }\end{array}$ parameter, $R a=\frac{\rho g \alpha \mathrm{dk}_{1}\left(T_{0}-T_{1}\right)}{\mu \kappa_{m}}, \quad$ is the thermal Rayleigh number, $R n=\frac{\left(\rho_{\mathrm{p}}-\rho\right) \varphi_{0} \mathrm{gk}_{1} \mathrm{~d}}{\mu \kappa_{\mathrm{m}}}$, the nanoparticle Rayleigh number,

$$
N_{A}=\frac{D_{T}\left(T_{0}-T_{1}\right)}{D_{B} T_{1} \varphi_{0}},
$$

$\mathrm{N}_{\mathrm{B}}=\frac{\varepsilon(\rho \mathrm{c})_{\mathrm{p}} \varphi_{0}}{(\rho \mathrm{c})_{\mathrm{f}}}$, the modified particle- density ratio, is the modified diffusivity ratio, modified Taylor number and $\mathrm{R}_{e a}=\frac{\gamma^{2} K E_{0}^{2} d^{2}(\Delta \mathrm{T})^{2}}{\mu \kappa_{\mathrm{m}}}$, is the AC electric Rayleigh number.

The dimensionless boundary conditions are

$$
\begin{aligned}
& w=\frac{\partial^{2} w}{\partial z^{2}}=\frac{\partial K}{\partial z}=0, T=1, \frac{\partial \phi}{\partial z}+N_{A} \frac{\partial T}{\partial z}=0 \text { at } z=0 \\
& \text { and } w=\frac{\partial^{2} w}{\partial z^{2}}=\frac{\partial K}{\partial z}=0, T=0, \frac{\partial \phi}{\partial z}+N_{A} \frac{\partial T}{\partial z}=0 \text { at } z=1
\end{aligned}
$$




\subsection{Basic Solutions}

We assume that the basic state is quiescent [Nield and Kuznetsov (2009), Sheu (2011), Chand and Rana (2012), Nield and Kuznetsov (2014)] and is given by

$$
\begin{gathered}
u=v=w=0, P=P(z), \mathrm{K}=\mathrm{K}_{\mathrm{b}}(\mathrm{z}), T=T_{b}(z), \varphi=\varphi_{b}(z), E=E_{b}(z), \quad \psi=\psi_{b}(\mathrm{z}) . \\
T_{b}=T_{0}-\frac{\Delta T}{d} z, \phi_{b}=\phi_{0}+\left(\frac{D_{T} \Delta T}{D_{B} T_{1} d}\right) z, K_{b}=K_{0}\left(1+\frac{\gamma \Delta T}{d} z\right) \hat{k}, E_{b}=\frac{E_{0}}{1+\frac{\gamma \Delta T}{d} z} \hat{k}
\end{gathered}
$$

Also we have

$$
\mathbf{V}_{\mathrm{b}}(z)=-\frac{E_{0} d}{\gamma \Delta T} \log \left(1+\frac{\gamma \Delta T}{d}\right) \hat{k}
$$

where $E_{0}=-\frac{V_{1} \frac{\gamma \Delta T}{d}}{\log (1+\gamma \Delta T)}$ is the root mean square value of the electric field at $\mathrm{z}=0$.

The basic state defined in (19) is substituted into equations (18) and (19), these equations reduce to

$$
\begin{gathered}
\frac{d^{2} \varphi_{b}(z)}{d z^{2}}+N_{A} \frac{d^{2} T_{b}(z)}{d z^{2}}=0, \\
\frac{d^{2} T_{b}(z)}{d z^{2}}+\frac{N_{B}}{L e} \frac{d \varphi_{b}(z)}{d z} \frac{d T_{b}(z)}{d z}+\frac{N_{A} N_{B}}{L e}\left(\frac{d T_{b}(z)}{d z}\right)^{2}=0,
\end{gathered}
$$

Using boundary conditions (18) in equations (15) and (16), on integration equation (20) gives

$$
\frac{d \varphi_{b}(z)}{d z}+N_{A} \frac{d T_{b}(z)}{d z}=0
$$

Using equation (22) in (21), we obtain

$$
\frac{d^{2} T_{b}(z)}{d z^{2}}=0
$$

Applying the boundary conditions (18), the solution of equation (23) is given by

$$
T_{b}(z)=1-z
$$

Integrating equation (22) by applying the boundary conditions (18), we get

$$
\varphi_{b}(z)=\phi_{0}+N_{A} z
$$

These results are identical with the results obtained by Sheu (2011) and Nield and Kuznetsov (2009).

\subsection{Perturbation Solutions}

To study the stability of the system, we superimposed infinitesimal perturbations on the basic state, so that 


$$
\mathbf{q}(u, v, w)=q^{\prime \prime}(u, v, w), T=T_{b}+T^{\prime \prime}, \mathrm{K}=\mathrm{K}_{\mathrm{b}}+\mathrm{K}^{\prime \prime}, p=p_{b}+p^{\prime \prime}, \mathrm{E}=E_{b}+E^{\prime \prime}+V=V_{b}+\mathrm{V}^{\prime \prime} .
$$

Introducing equation (26) into equations (13) - (17), linearizing the resulting equations by neglecting nonlinear terms that are product of prime quantities and dropping the primes (") for convenience, the following equations are obtained

$$
\begin{gathered}
\nabla \cdot \mathbf{q}=0, \\
0=-\nabla P-\left(1-F \frac{\partial}{\partial t}\right) \mathbf{q}+\operatorname{RaT} \hat{e}_{z}-\operatorname{Rn} \varphi \hat{e}_{z}+\sqrt{T a}\left(v \hat{e}_{x}-\hat{e}_{y}\right)+R_{e a}\left(T-\frac{\partial K}{\partial z}\right), \\
\frac{1}{\sigma} \frac{\partial \varphi}{\partial t}+\frac{1}{\varepsilon} w=\frac{1}{L e} \nabla^{2} \varphi+\frac{N_{A}}{L e} \nabla^{2} T, \\
\frac{\partial T}{\partial t}-w=\nabla^{2} T+\frac{N_{B}}{L e}\left(N_{A} \frac{\partial T}{\partial z}-\frac{\partial \varphi}{\partial z}\right)-\frac{2 \mathrm{~N}_{A} N_{B}}{L e} \frac{\partial T}{\partial z} . \\
\nabla^{2} V=\frac{\partial T}{\partial z} .
\end{gathered}
$$

Operating equation (28) with $\hat{e}_{z} \cdot$ curl curl to eliminate the pressure term $P$, we obtain

$$
0=-\left(1-F \frac{\partial}{\partial t}\right) \nabla^{2} w+R a \nabla_{H}^{2} \mathrm{~T}-\mathrm{Rn}_{H}^{2} \varphi-\sqrt{T a} \frac{\partial \xi}{\partial z}+R_{e a} \nabla_{H}^{2}\left(T-\frac{\partial K}{\partial z}\right),
$$

$\nabla_{H}^{2}=\frac{\partial^{2}}{\partial x^{2}}+\frac{\partial^{2}}{\partial y^{2}}$. is the two-dimensional Laplace operator on the horizontal plane and $\xi=\frac{\partial v}{\partial x}-\frac{\partial u}{\partial y}$ is the vorticity.

Again eliminating pressure $P$ from Eq. (28) and introducing vorticity $\xi$, we get

$$
\xi=\sqrt{T a} \frac{\partial w}{\partial z}
$$

Now, eliminating $\xi$ between Eqs. (28) and (29), we obtain

$$
0=-\left(\left(1-F \frac{\partial}{\partial t}\right) \nabla^{2}+T a \frac{\partial^{2}}{\partial z^{2}}\right) w+R a \nabla_{H}^{2} \mathrm{~T}-\operatorname{Rn} \nabla_{H}^{2} \varphi-\sqrt{T a} \frac{\partial \xi}{\partial z}+R_{e a} \nabla_{H}^{2}\left(T-\frac{\partial K}{\partial z}\right)
$$

Boundary conditions are

$$
w=\frac{\partial^{2} w}{\partial z^{2}}=\frac{\partial V}{\partial z}=0, T=0, \frac{\partial \phi}{\partial z}+N_{A} \frac{\partial T}{\partial z}=0 \text { at } z=0 \text { and } \text { at } z=1
$$

\section{Normal Mode Analysis}

We express the disturbances into normal modes of the form

$$
[w, T, \varphi, V]=[W(z), \Theta(z), \Phi(z), \Psi(\mathrm{z})] \exp (i l x+i m y+\omega t),
$$


where $l, m$ are the wave numbers in the $x$ and $y$ direction, respectively, and $\omega$ is the growth rate of the disturbances.

Substituting equation (36) into equations (27) - (31) and (34), we obtain the following eigenvalue problem

$$
\begin{gathered}
\left(\left(D^{2}-a^{2}\right)(1-\omega F)+T a D^{2}\right) W+a^{2} R a \Theta-a^{2} R n \Phi+a^{2} R_{e a}(\Theta-D \Psi)=0, \\
W+\left(D^{2}+\frac{N_{A}}{L e} D-\frac{2 N_{A} N_{B}}{L e} D-a^{2}-\omega\right) \Theta-\frac{N_{B}}{L e} D \Phi=0, \\
\frac{1}{\varepsilon} W-\frac{N_{A}}{L e}\left(D^{2}-a^{2}\right) \Theta-\left(\frac{1}{L e}\left(D^{2}-a^{2}\right)-\frac{\omega}{\sigma}\right) \Phi=0 \\
\left(D^{2}-a^{2}\right) \Psi=D \Theta
\end{gathered}
$$

where $D=\frac{d}{d z}$ and $a^{2}=l^{2}+m^{2}$ is the dimensionless resultant wave number.

The boundary conditions of the problem in view of normal mode analysis are

$$
W=0, D^{2} W=0, \Theta=0, D \Psi=0, D \Phi+N_{A} D \Theta=0 \quad \text { at } z=0 \text { and } z=1 \text {. }
$$

\section{Method of solution}

The Galerkin-type weighted residuals method is used to find an approximate solution of the system of equations (37) - (40) with the corresponding boundary conditions (41). In this method, the test functions are the same as the base (trial) functions. Thus, we can write

$$
W=\sum_{s=1}^{N} A_{s} W_{s}, \Theta=\sum_{s=1}^{N} B_{s} \Theta_{s}, \Phi=\sum_{s=1}^{N} C_{s} \Phi_{s}, \Psi=\sum_{s=1}^{N} D_{s} \Psi_{s},
$$

where $A_{s}, B_{s}, C_{s}$ and $D_{s}$ are unknown coefficients, $\mathrm{s}=1,2,3, \ldots \ldots, \mathrm{N}$ and the base functions $W_{s}$, $\Theta_{s}, \Phi_{s}$ and $\Psi_{s}$ satisfy the boundary conditions (41). Using expression for $W, \Theta, \Phi$ and $\Psi$ in equations (39) - (40) and multiplying the first equation by $W_{s}$, second by $\Theta_{s}$, third by $\Phi_{s}$ and fourth by $\Psi_{s}$; then integrating between the limits 0 to 1 , we obtain a set of $3 \mathrm{~N}$ homogeneous equations with $3 \mathrm{~N}$ unknowns $A_{s}, B_{s}, C_{s}$ and $D_{s} ; s=1,2,3, \ldots, \mathrm{N}$. For the existence of non-trivial solution, the vanishing of the determinant of coefficients produces the characteristics equation of the system in terms of Rayleigh number $R a$.

\section{Linear Stability Analysis and Dispersion Relation}

We have considered the case of free boundaries for which system of Eqs. (37) - (40) together with the boundary conditions (41) constitute a linear eigenvalue problem with variable coefficient for the growth rate of disturbance of the system. The resulting eigenvalue problem is solved numerically by the Galerkin method of first order $(\mathrm{N}=1)$, which gives the expression for Rayleigh number $\mathrm{Ra}$ as

Substituting Eq. (42) into Eqs. (37) - (40), we obtain the following matrix equation 


$$
\left[\begin{array}{cccc}
\left(\pi^{2}+a^{2}\right)(1-F \omega)+\pi^{2} T a & -a^{2}\left(R a+R_{e a}\right) & -a^{2} N_{A} R n & -a^{2} \pi R_{e a} \\
1 & -\left(\pi^{2}+a^{2}+\omega\right) & 0 & 0 \\
\frac{1}{\varepsilon} & \frac{1}{L e}\left(\pi^{2}+a^{2}\right) & -\left(\frac{1}{L e}\left(\pi^{2}+a^{2}\right)+\frac{\omega}{\sigma}\right) & 0 \\
0 & -\pi & 0 & -\left(\pi^{2}+a^{2}\right)
\end{array}\right]\left[\begin{array}{l}
W \\
\Theta \\
\Phi \\
\Psi
\end{array}\right]=\left[\begin{array}{l}
0 \\
0 \\
0 \\
0
\end{array}\right]
$$

The linear system (43) has a non-trivial solution if and only if

$$
\mid\left[\begin{array}{cccc}
\left(\pi^{2}+a^{2}\right)(1-F \omega)+\pi^{2} T a & -a^{2}\left(R a+R_{e a}\right) & -a^{2} N_{A} R n & -a^{2} \pi R_{e a} \\
1 & -\left(\pi^{2}+a^{2}+\omega\right) & 0 & 0 \\
\frac{1}{\varepsilon} & \frac{1}{L e}\left(\pi^{2}+a^{2}\right) & -\left(\frac{1}{L e}\left(\pi^{2}+a^{2}\right)+\frac{\omega}{\sigma}\right) & 0 \\
0 & -\pi & 0 & -\left(\pi^{2}+a^{2}\right)
\end{array}\right] \|=0
$$

which yields

$$
\begin{gathered}
R a=\frac{\left((1-\omega F)\left(\pi^{2}+a^{2}\right)+\pi^{2} T a\right)\left(\pi^{2}+a^{2}+\omega\right)}{a^{2}}-\frac{a^{2}}{\left(\pi^{2}+a^{2}\right)} R_{e a} \\
-\frac{\varepsilon N_{A}\left(\pi^{2}+a^{2}\right)+L e\left(\pi^{2}+a^{2}+\omega\right)}{\left(\pi^{2}+a^{2}\right) \sigma+\omega L e} \frac{\sigma}{\varepsilon} R n
\end{gathered}
$$

Equation (44) is the required dispersion relation accounting for the effect of rotation, Lewis number, kinematic visco-elasticity parameter, AC electric Rayleigh number, nanoparticle Rayleigh number, modified diffusivity ratio on thermal instability in a layer of Walters' (model B') elastico-viscous nanofluid saturating a porous medium under vertical AC electric field.

For neutral stability, the real part of $\omega$ is zero. Hence on putting $\omega=i \omega$, (where $\omega$ is real and is dimensionless frequency) in Eq. (44), we have

$$
R a=\Delta_{1}+i \omega \Delta_{2}
$$

where

$$
\Delta_{1}=\frac{\left(\pi^{2}+a^{2}\right)\left(\pi^{2}+a^{2}+\pi^{2} T a+\omega^{2} F\right)}{a^{2}}-\frac{a^{2}}{\left(\pi^{2}+a^{2}\right)} R_{e a}-\frac{\left(\pi^{2}+a^{2}\right)^{2}\left(N_{A}+\frac{L e}{\varepsilon}\right)+\frac{\omega^{2}}{\sigma \varepsilon}}{\left(\pi^{2}+a^{2}\right)^{2}+\left(\frac{\omega L e}{\sigma}\right)^{2}} R n
$$

and

$$
\Delta_{2}=\frac{\left(\pi^{2}+a^{2}\right)\left(1-F\left(\pi^{2}+a^{2}\right)\right)+\pi^{2} T a}{a^{2}}+\frac{\left(\pi^{2}+a^{2}\right)\left(\frac{L e}{\sigma}\left(N_{A}+\frac{L e}{\varepsilon}\right)-\frac{L e}{\varepsilon}\right)+\frac{\omega^{2}}{\sigma \varepsilon}}{\left(\pi^{2}+a^{2}\right)^{2}+\left(\frac{\omega L e}{\sigma}\right)^{2}} R n
$$


Since $R a$ is a physical quantity, so it must be real. Hence, it follows from the Eq. (44) that either $\omega=0$ (exchange of stability, steady state) or $\Delta_{2}=0(\omega \neq 0$ overstability or oscillatory onset).

\section{Stationary Convection}

Since oscillatory convection has been ruled out, because of the absence of two opposing buoyancy forces, we need to consider only the case of stationary convection. Put $\omega=0$ in equation (44), we obtain

$$
R a=\frac{\left(\pi^{2}+a^{2}\right)^{2}+\left(\pi^{2}+a^{2}\right) \pi^{2} T a}{a^{2}}-\frac{a^{2}}{\left(\pi^{2}+a^{2}\right)} R_{e a}-\left(N_{A}+\frac{L e}{\varepsilon}\right) R n .
$$

Equation (48) expresses the Rayleigh number as a function of the dimensionless resultant wave number $a$ and the different parameters $T a, R_{e a}, \varepsilon, R n, L e, N_{A}$. Since the elastico-viscous parameter $F$ vanishes with $\omega$ so the Walters' (model B') elastico-viscous nanofluid fluid behaves like an ordinary Newtonian nanofluid. Equation (48) is identical to that obtained by Kuznetsov and Nield (2009) and Rana and Chand (2016). Also, in equation (48) the particle increment parameter $N_{B}$ does not appear and the diffusivity ratio parameter $N_{A}$ appears only in association with the nanoparticle Rayleigh number $R n$. This implies that the nanofluid cross-diffusion terms approach to be dominated by the regular cross-diffusion term.

In the absence of $\mathrm{AC}$ electric field Rae, equation (48) reduces to

$$
R a=\frac{\left(\pi^{2}+a^{2}\right)^{2}+\left(\pi^{2}+a^{2}\right) \pi^{2} T a}{a^{2}}-\left(N_{A}+\frac{L e}{\varepsilon}\right) R n .
$$

which is identical with the result derived by Kuznetsov and Nield (2009), Rana et al. (2015) and Rana and Chand (2016).

In the absence of rotation, equation (49) becomes

$$
R a=\frac{\left(\pi^{2}+a^{2}\right)^{2}}{a^{2}}-\left(N_{A}+\frac{L e}{\varepsilon}\right) R n .
$$

To study the effect of AC electric Rayleigh number, Lewis number, nanoparticle Rayleigh number, modified diffusivity ratio and medium porosity, we examine the behavior of $\frac{\partial R a}{\partial T a}, \frac{\partial R a}{\partial R_{e a}}, \frac{\partial R a}{\partial L e}, \frac{\partial R a}{\partial N_{A}}, \frac{\partial R a}{\partial R n}$ and $\frac{\partial R a}{\partial \varepsilon}$ analytically.

From equation (48), we obtain

$$
\begin{gathered}
\frac{\partial R a}{\partial T a}=\frac{\left(\pi^{2}+a^{2}\right) \pi^{2}}{a^{2}} \\
\frac{\partial R a}{\partial R_{e a}}=-\frac{a^{2}}{\left(\pi^{2}+a^{2}\right)} \\
\frac{\partial R a}{\partial L e}=-\frac{R n}{\varepsilon}
\end{gathered}
$$




$$
\begin{gathered}
\frac{\partial R a}{\partial N_{A}}=-R n \\
\frac{\partial R a}{\partial R n}=-\left(N_{A}+\frac{L e}{\varepsilon}\right) \\
\frac{\partial R a}{\partial \varepsilon}=\frac{L e R n}{\varepsilon^{2}}
\end{gathered}
$$

\section{Results and Discussions}

The thermal Rayleigh number on the onset of stationary convection is given by (48) which does not depend on viscoelastic parameter. It takes the same value that the one obtained for an ordinary Newtonian fluid. According to the definition of nanoparticle Rayleigh number $R n$ in Eq. (25), this corresponds to negative value of Rn for bottom-heavy distribution of nanoparticles $\left(\rho_{p}>\rho\right)$. In such cases, values of $N_{A}$ are also negative as defined in the paper. In the following discussion, negative values of $R n$ and $N_{A}$ are presented.

From equation (51), we see that the partial derivative of Rayleigh number $R a$ with respect to modified Taylor number $T a$ is positive implying thereby rotation stimulates the stationary convection. Thus, rotation parameter has stabilizing effect on the system for both top-heavy and bottom-heavy nanoparticle distribution which is in an agreement with the result derived by Shivakumara (2011) and Chand et al. (2017).

The right hand sides of equations (52) and (55) are negative implying, thereby the AC electric Rayleigh number and nanoparticle Rayleigh number inhibit the stationary convection. Thus, AC electric Rayleigh number and nanoparticle Rayleigh number have destabilizing effects on the system which is in an agreement with the results derived by Nield and Kuznetsov (2009), Takashima (1976) and Shivakumara (2011), Rana et al. (2012, 2015) and Chand et al. (2017).

The right hand sides of equations (53) and (54) are negative if nanoparticle Rayleigh number Rn is positive but for the bottom-heavy nanoparticle distribution, $\mathrm{Rn}$ is negative. Thus, Lewis number Le and modified diffusivity ratio $\mathrm{N}_{\mathrm{A}}$ have stabilizing effect on the system for bottom-heavy nanoparticle distribution which is in an agreement with the result obtained by Sheu (2011).

The right-hand side of equation (56) is positive but it will be negative if $\mathrm{Rn}$ is negative implying thereby medium porosity has destabilizing effect on the system which is in an agreement with the results derived by Nield and Kuznetsov (2009), Rana et al. (2012, 2015) and Chand et al. (2017).

The dispersion relation (48) is also analyzed numerically. Graphs have been plotted by giving some numerical values to the parameters to depict the stability characteristics, e. g., $10^{2} \leq L e \leq 10^{4}$ (Lewis number), $10^{-1} \leq R n \leq 10$ (nanoparticles Rayleigh number), $0.1 \leq \varepsilon \leq 1$ (porosity parameter) (Rana et al. 2012, 2015) and $10 \leq \operatorname{Re} \leq 10^{4}$ (AC electric Rayleigh number), $10 \leq T a \leq 10^{4}$ (Shivakumara et al. 2011). Stability curves for modified Taylor number Ta, AC electric Rayleigh number $R_{e a}$, Lewis number $L e$, nanoparticles Rayleigh number $R n$, modified diffusivity ratio $N_{A}$ and porosity parameter $\varepsilon$ are shown in Figures 2-7. 


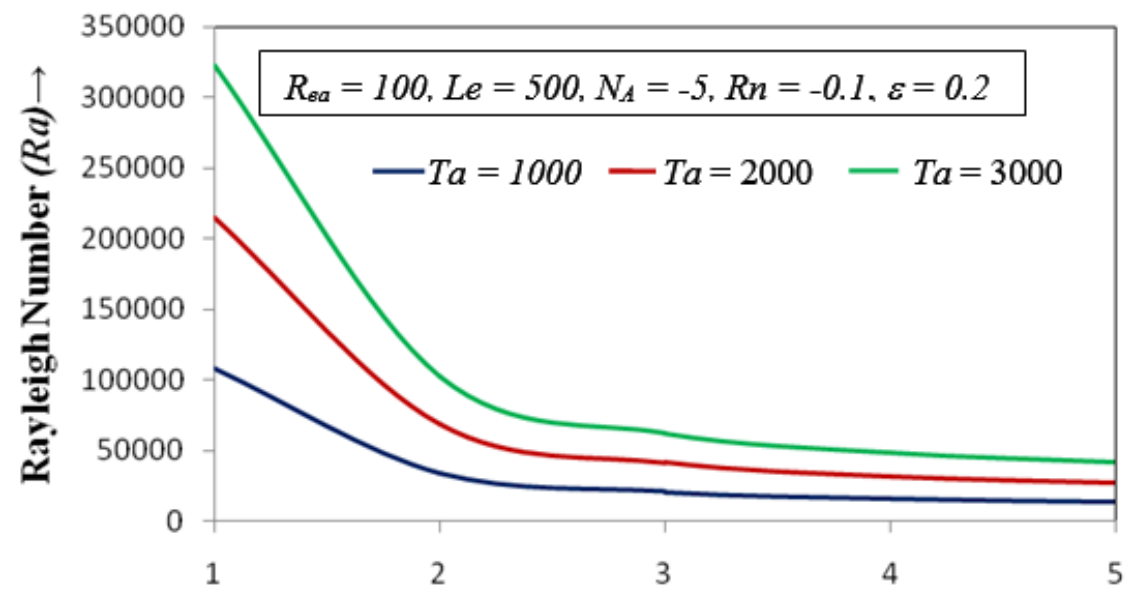

Wave Number $(a) \rightarrow$

Fig. 2. The variations of thermal Rayleigh number $R a$ with the wave number $a$ for different values of the modified Taylor number $T a=1000, T a=2000$ and $T a=3000$.

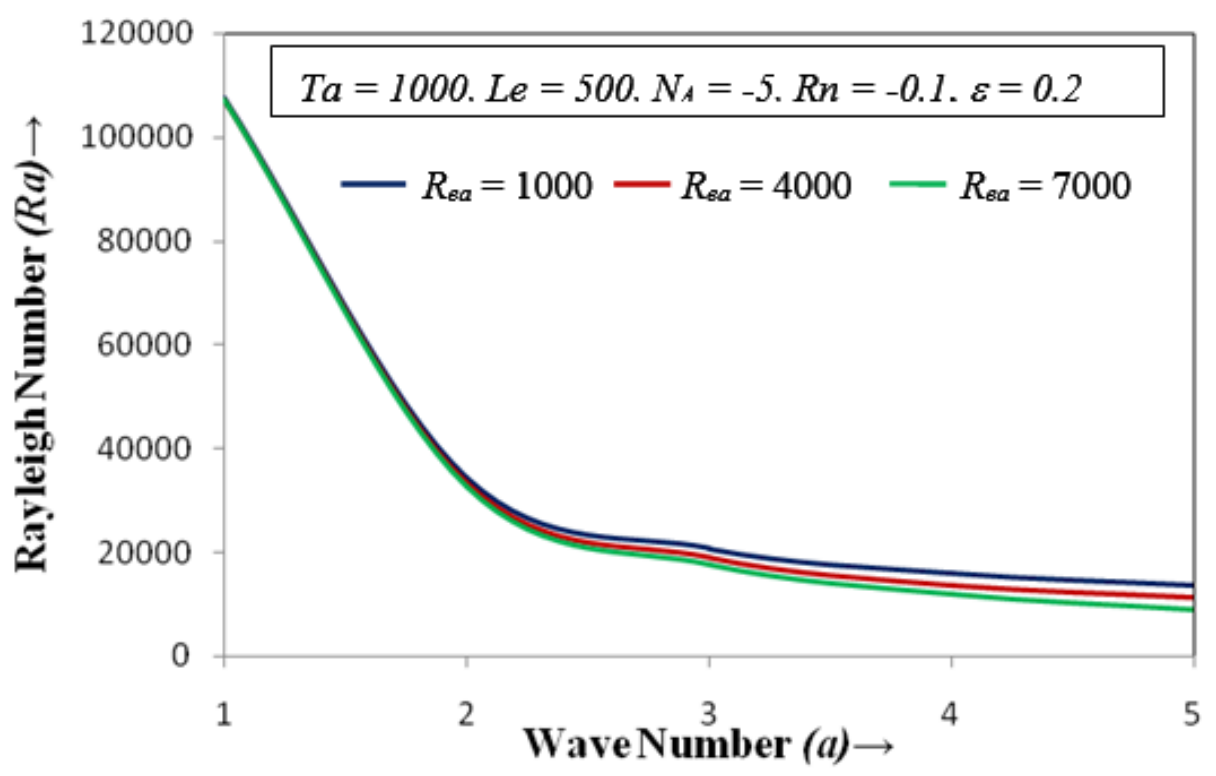

Fig. 3. The variations of thermal Rayleigh number $R a$ with the wave number $a$ for different values of the AC electric Rayleigh number Rea $=1000$, Rea $=4000$ and Rea $=7000$. 


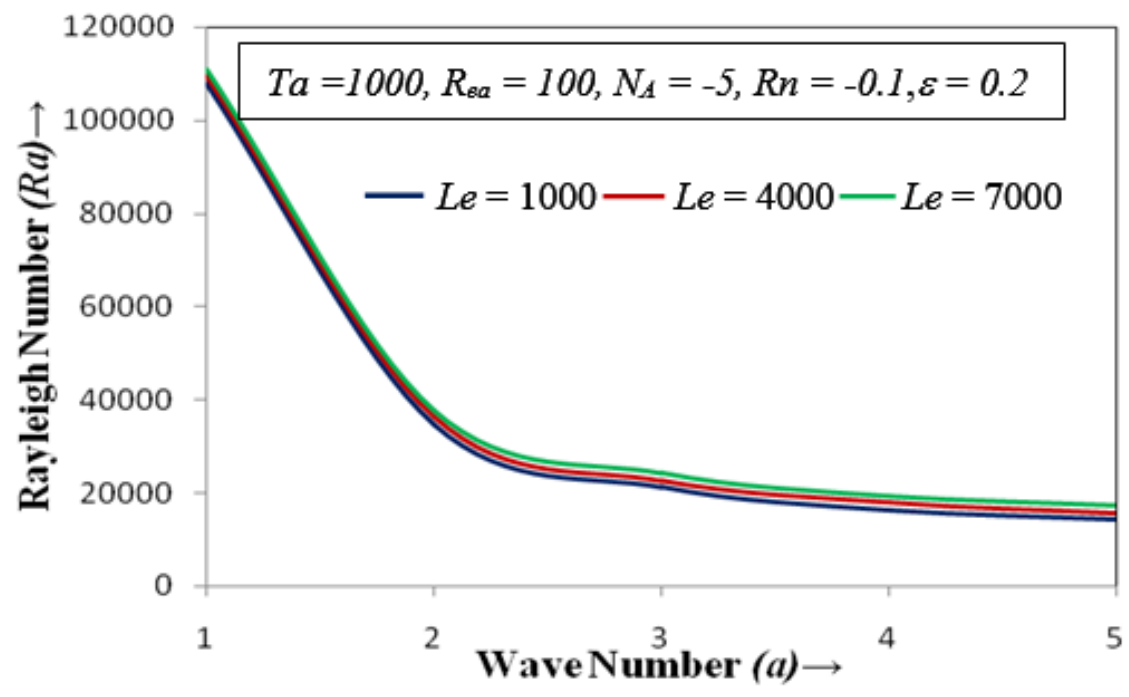

Fig. 4. The variations of thermal Rayleigh number $R a$ with the wave number $a$ for different values of the Lewis number $L e=1000, \mathrm{Le}=4000, \mathrm{Le}=7000$.

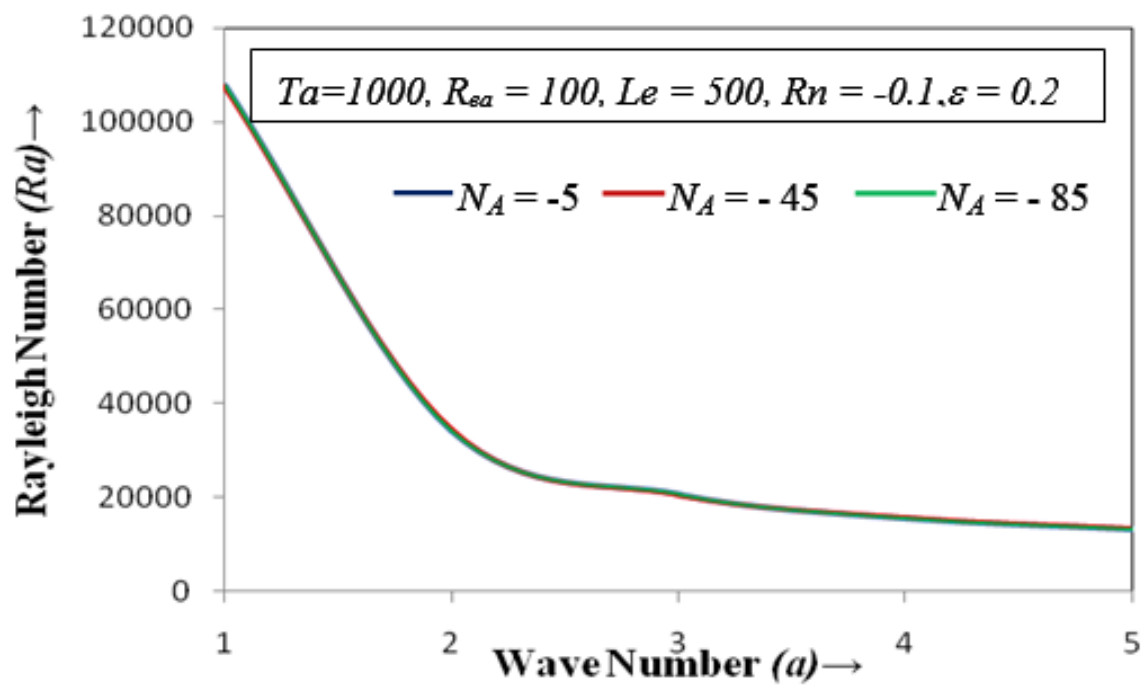

Fig. 5. The variations of thermal Rayleigh number $R a$ with the wave number $a$ for different values of the modified diffusivity ratio $N_{A}=-5, N_{A}=-45$ and $N_{A}=-85$. 


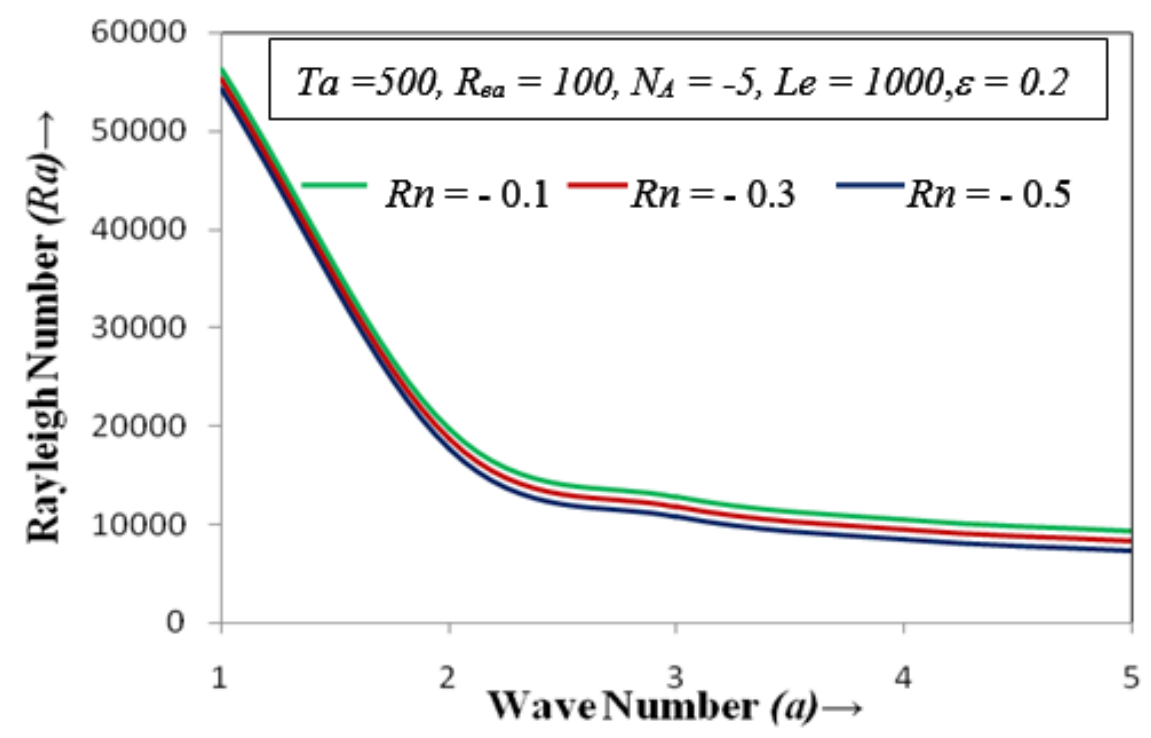

Fig. 6. The variations of thermal Rayleigh number $R a$ with the wave number $a$ for different values of the nanoparticle Rayleigh number $R n=-0.1, R n=-0.3, R n=-0.5$.

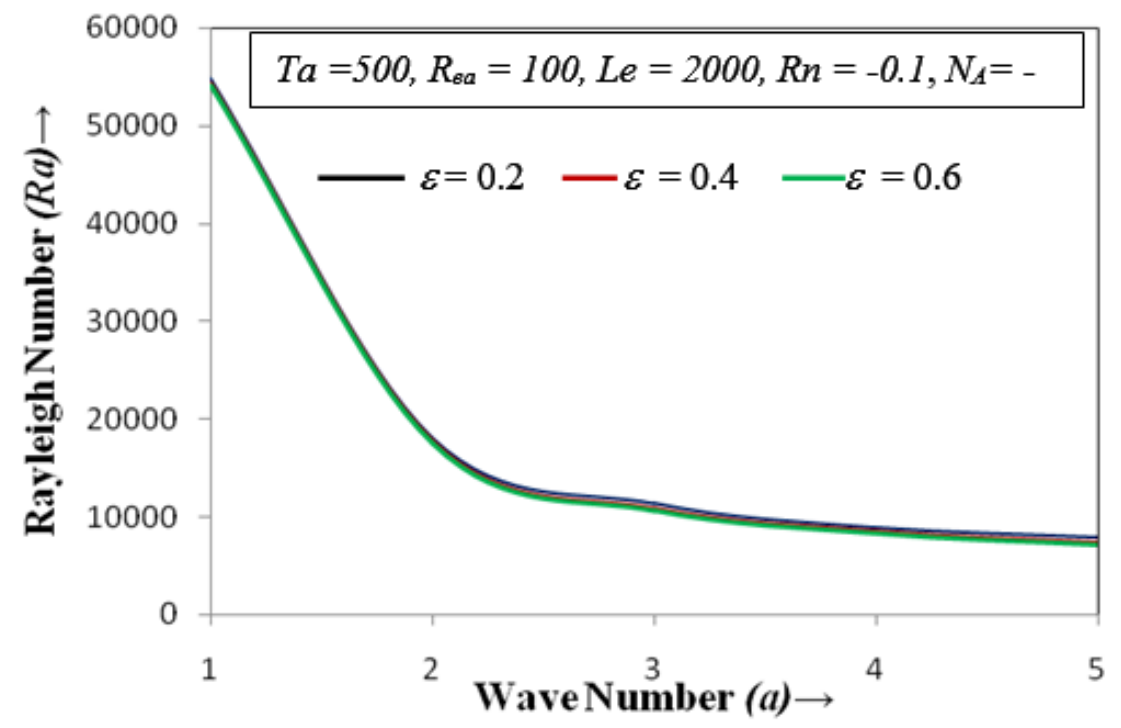

Fig.7. The variations of thermal Rayleigh number $R a$ with the wave number $a$ for different values of the medium porosity $\varepsilon=0.2, \varepsilon=0.4$ and $\varepsilon=0.6$.

In Fig. 2, the variations of thermal Rayleigh number $R a$ with the wave number $a$ for three different values of the modified Taylor number, namely, $T a=1000,2000$ and 3000 which shows that thermal Rayleigh number increases with the increase in the modified Taylor number. Thus, rotation parameter has stabilizing effect on the system. The variations of thermal Rayleigh number $R a$ with the wave number a for three different values of the AC electric Rayleigh number, namely, Rea $=1000,4000$ and 7000 is plotted in Fig. 3 and it is observed that the thermal 
Rayleigh number decreases with the increase in AC electric Rayleigh number implying there by AC electric Rayleigh number destabilizes the system. In Fig. 4, the variations of thermal Rayleigh number $R a$ with the wave number a for three different values of the nanofluid Lewis number, namely, $L e=1000,4000$ and 7000 which shows that thermal Rayleigh number increases with the increase in Lewis number. Thus, Lewis number has stabilizing effect on the system.

The variations of thermal Rayleigh number $R a$ with the wave number $a$ for three different values of the modified diffusivity ratio, namely, $\mathrm{N}_{\mathrm{A}}=-5,-45,-85$ is plotted in Fig. 5 and it is found that thermal Rayleigh number increases slightly with the increase in modified diffusivity ratio implying thereby modified diffusivity ratio has very little stabilizing effect on the system. For negative values of $N A$, an increase of $N_{A}$ reduces the thermophoresis effect of pushing the heavier nanoparticles upwards. As a result, the stabilizing effects of particle distributions enhanced. Thus, the effect of increasing $N_{A}$ is to stabilize the system when $R n$ is negative.

In Fig. 6, the variations of thermal Rayleigh number $R a$ with the wave number $a$ for three different values of the nanoparticle Rayleigh number, namely $R n=-0.2,-0.4,-0.6$ which shows that thermal Rayleigh number increases with the increase in nanoparticle Rayleigh number. Thus nanoparticle Rayleigh number has a stabilizing effect on the system. The variations of thermal Rayleigh number $R a$ with the wave number $a$ for three different values of medium porosity, namely $\varepsilon=0.2,0.4$ and 0.6 is plotted in Fig. 7 and it is found that thermal Rayleigh number decreases slightly with the increase in medium porosity implying thereby medium porosity has a very low destabilizing effect on the onset of stationary convection in a layer of Walters' (model B') elastico-viscous nanofluid saturating a porous medium.

\section{Conclusion}

On the onset of electrohydrodynamic thermal instability in a layer of Walters' (model B') elastic viscous Nanofluid under rotation saturating a porous medium has been investigated by using a linear stability analysis and Galerkin method. The elastico-viscous nanofluid incorporates the Brownian motion and thermophoresis. For the case of stationary convection, the Walters' (model B') nanofluid behaves like an ordinary Newtonian nanofluid. Kinematic viscoelasticity has no effect on the onset of stationary convection. It is found that the Taylor number, Lewis number and nanoparticles Rayleigh number has stabilizing effect whereas the AC electric field Rayleigh Number has destabilizing effect on the stationary convection. Modified diffusivity ratio and medium porosity have slightly destabilizing effect. Oscillatory convection has been ruled out under the considered boundary conditions.

\section{References}

Buongiorno J (2006). Convective transport in nanofluids, ASME Journal of Heat Transfer 128: 240-250.

Castellanos A (1998). Electrohydrodynmics, New York: Springer-Verlag Wien New York.

Chand R, Rana GC (2012). Thermal instability of Rivlin-Ericksen elastico-viscous nanofluid saturated by a porous medium, J. Fluids Eng. 134: 121203.

Chand R., Kango SK, Rana GC (2014). Thermal instability in anisotropic porous medium saturated by a nanofluid-a realistic approach, NSNTAIJ 8: 445-453.

Chand R, Rana GC, Yadav D (2017). Thermal instability in a layer of couple stress nanofluid saturated porous medium, J. of Theoretical and Applied Mechanics, Sofia 47: 69-84, 
Chand R, Rana GC (2017). Thermal instability of maxwell visco-elastic nanofluid in a porous medium with thermal conductivity and viscosity variation, Structural Integrity and Life, 17: 113-120.

Choi S (1995). Enhancing thermal conductivity of fluids with nanoparticles. In: Siginer D.A., Wang, H.P. (eds.) Developments and Applications of Non-Newtonian Flows, ASME FEDVol. 231/MD-Vol. 66: 99-105.

Chandrasekhar S (1961). Hydrodynamic and Hydromagnetic Stability, Dover Publication, New York.

Gupta U, Aggarwal P (2011). Thermal instability of compressible Walters' (model B') fluid in the presence of hall currents and suspended particles, Thermal Science 15: 487-500.

Ingham DD, Pop I (1981). Transport Phenomena in Porous Media, Elsvier, New York.

Jones TB (1978). Electrohydrodynamically enhanced heat transfer in liquids-A review, In T.F. Irvine Jr. \& J.P.

Hartnett (Eds.), Advances in Heat Transfer. Academic Press, pp. 107-144. Kuznetsov AV, Nield DA (2009). Thermal instability in a porous medium layer saturated by a nanofluid: Brinkman Model, Transp. Porous Medium 81: 409-422. DOI 10.1007/s11242-009-9413-2

Landau LD (1960). Electrodynamics of Continuous Media, New York: Oxford.

Melcher JR, Taylor GI (1969a). Electrohydrodynamics: a review of the role of interfacial shear stresses, Annu. Rev. Fluid Mech. 1: 11-146.

Nield DA, Bejan A (2006). Convection in Porous Medium, Springer, New York.

Nield DA, Kuznetsov AV (2009). Thermal instability in a porous medium layer saturated by a nanofluid, Int. J. Heat Mass Transf. 52: 5796-5801.

Nield DA, Kuznetsov AV (2014). Thermal instability in a porous medium layer saturated by a nanofluid: a revised model, Int. J. Heat Mass Transf. 68: 211-214.

Othman MI (2004). Electrohydrodynamic instability of a rotating layer of a viscoelastic fluid heated from below, Zeitschriftfür Angewandte Mathematik und Physik 55: 468-482. DOI 10.1007/s00033-003-1156-2.

Rana GC, Kango SK, Kumar S (2012). Effect of Rotation on the onset of convection in Walters' (model B') heated from below in a Brinkman porous medium, J. of Porous Media 15: 11491153. DOI: 10.1615/JPorMedia.v15.i12.70

Rana GC, Chand R, Yadav D (2015). The onset of electrohydrodynamic instability of an elasticoviscous Walters' (Model B') dielectric fluid layer, FME Transactions, 43: 154-160. DOI: $10.5937 /$ fmet1502154R

Rana GC, Chand R, Sharma V (2016). On the onset of instability of a viscoelastic fluid saturating a porous medium in electrohydrodynamics, Rev. Cub. Fis. 33, 89-94.

Roberts PH (1969b). Electrohydrodynamic convection, Quart. J. of Mech. and Appl. Math. 2: 211-220.

Tzou DY (2008). Instability of nanofluids in natural convection, ASME J. Heat Transf. 130: 072401.

Ruo AC, Chang, MH F Chen (2010). Effect of rotation on the electrohydrodynamic instability of a fluid layer with an electrical conductivity gradient, Physics of Fluids 22: 024102-1024102-11.

Sharma V, Rana GC (2001). Thermal instability of a Walters' (Model B') elastico-viscous fluid in the presence of variable gravity field and rotation in porous medium, J. Non-Equilib. Thermodyn. 26: 1-40.

Sheu LJ (2011). Thermal instability in a porous medium layer saturated with a viscoelastic nanofluid, Transp. Porous Med., 88: 461-477. DOI 10.1007/s11242-011-9749-2

Shivakumara IS, Lee J, Malashetty MS, Sureshkumara S (2011). Effect of thermal modulation on the onset of thermal convection in Walters' B viscoelastic fluid in a porous medium, Transport in Porous Media 87: 291-307. DOI 10.1007/s11242-010-9682-9 
Shivakumara IS, Nagashree MS, Hemalatha K (2007). Electroconvective instability in a heat generating dielectric fluid layer, Int. Comm. in Heat and Mass Transfer 34: 1041-1047. DOI: 10.1016/j.icheatmasstransfer.2007.05.006

Shivakumara IS, Akkanagamma M, Chiu-on Ng (2013). Electrohydrodynamic instability of a rotating couple stress dielectric fluid layer, Int. J. Heat Mass Transfer 62: 761-771.

Takashima M (1976). The effect of rotation on electrohydrodynamic instability, Canadian Journal of Physics 54: 342-347.

Takashima M, Ghosh .K (1979). Electrohydrodynamic instability in a viscoelastic liquid layer, $J$. Phys. Soc. Japan 47 : 1717-1722

Takashima M, Hamabata H (1984). The stability of natural convection in a vertical layer of dielectric fluid in the presence of a horizontal ac electric field, J. Phys. Soc. Japan 53: 17281736.

Tzou DY (2008). Thermal instability of nanofluids in natural convection, Int. J. of Heat and Mass Transfer 51: 2967-2979.

Vafai K, Hadim HA (2000). Hand Book of Porous Media, M. Decker, New York.

Walters' K (1962). The solution of flow problems in the case of materials with memory, J. Mecanique, 2: 469-478.

Yadav D, Kim, MC (2015). The onset of transient Soret-driven buoyancy convection in a nanoparticles suspension with particle concentration-dependent viscosity in a porous medium, Journal of Porous Media 18: 369-378. DOI: 10.1615/JPorMedia.v18.i4.10 